\title{
Effects of stimulus rotation on discrimination learning by monkeys '
}

LESLIE H. HICKS HOWARD UNIVERSITY, WASHINGTON, D. C.

Monkey performance on object and pattern discriminations was compared under various stimulus rotational conditions: (1) positive and negative stimuli remain constant, (2) only positive stimulus rotates, (3) only negative stimulus rotates, (4) both stimuli rotate. Performance was best at the condition in which only the negative stimulus rotated.

The aim of these experiments was to measure monkey performance on object and pattern discrimination problems under various conditions of positional rotation of the stimuli. The ability of animals to transfer responses learned to a particular stimulus in one orientation to the same stimulus in a different orientation has been investigated in several species (raccoons, cats, rhesus, chimpanzees, and octopus -summarized in Sutherland, 1961). In general, the higher the organism on the phyletic scale, the better the performance. The specific conditions of the present experiment also permitted an evaluation of reward and nonreward effects during the course of learning these discrimination problems. In a recent study (Gardner \& Coate, 1965), rats were found to perform better on a simultaneous pattern discrimination when only the negative ( - ) stimulus varied from trial to trial as compared to only the positive (+) stimulus changing.

\section{EXPERIMENT 1}

\section{Subjects}

The Ss were five rhesus monkeys at the University of Wisconsin Primate Laboratory who had participated in object and pattern discrimination studies.

Appasatus and Procedure

The stimuli were three-dimensional toys mounted on a $3 \times 3$ in., 1/4 in. thick plywood base. Depending on the training condition, the stimuli were rotated through the following orientations: (1) standard or frontal view; (2) rotation of $90^{\circ}$ to the left; (3) rotation of $90^{\circ}$ to the right; (4) rotation of $180^{\circ}$ from the standard. Each $S$ received four 12-trial problems per day for 30 days. These consisted of one problem each under the following conditions of rotation:

Condition A: Both positive $(t)$ and negative $(-)$ stimuli remained in the standard orientation for all 12 trials. Condition B: The positive (t) stimulus was rotated through the four orientations from trial to trial with each orientation occurring three times per problem. The negative (-) stimulus remained in the same orientation for all trials.

Condition C: This condition was similar to Condition B, except that the negative ( - ) stimulus was rotated from trial to trial while the positive $(t)$ stimulus was not changed in orientation.
Table 1. Errors in object discrimination

\begin{tabular}{llr}
\multicolumn{1}{c}{ CONDITION } & Mn & \multicolumn{1}{c}{ SD } \\
\hline A. Both objects in standard orientation & 88 & 15.14 \\
B. Positive object rotates & 86.6 & 8.67 \\
C. Negative object rotates & 62.8 & 14.89 \\
D. Both objects rotate & 97.8 & 18.00 \\
\hline
\end{tabular}

Condition D: Both positive (+) and negative (-) stimuli were changed from trial to trial with three trials at each of the four orfentations.

The noncorrection procedure was used. A single raisin was the reward for a correct response and location of the stimuli on the two-hole board was determined by a Gellerman sequence. All testing occurred in the Wisconsin General Test Apparatus.

Results

Table 1 presents mean errors and standard deviations for the different rotational conditions.

After an analysis of variance of error scores in which blocks of day (learning) and rotation condition were significant $(F=7,48$, df $=5 / 60, p<.01$ and $F=11.13$, $d f=3 / 60, p<.01$, respectively), the means of the four rotation conditions were tested by the Duncan range procedure. Performance under the $\mathrm{C}$ condition (negative object rotates) was superior to that of each of the other conditions ( $p<.01)$. Condition B (positive object rotates) and Condition $A$ (both objects in standard orientation) performances were better than Condition $D$ performance $(p<.01)$. Figure 1 illustrates the course of learning under each of the conditions.

\section{EXPERIMENT 2}

\section{Subjects}

Four cynomologus and two rhesus monkeys served as Ss. All were experienced in object and pattern discrimination.

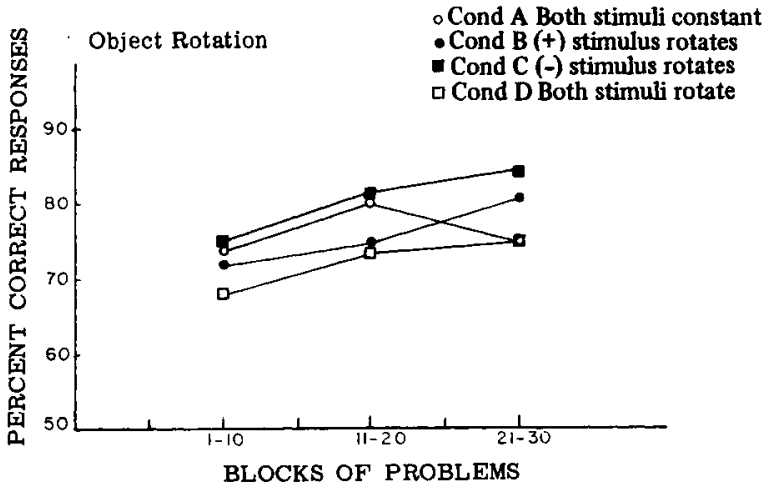

Fig. 1. Mean correct responses for blocks of problems under various stimulus object rotation conditions. 


\section{Apparatus and Procedure}

The pattern stimuli were India ink figures drawn on $3 \times 3$ in. poster board cards. The testing procedure was conducted in exactly the same manner as in Experiment 1.

Results

Means and standard deviations of error scores are presented in Table 2.

Blocks of problems and rotation conditions were significant $(F=5.88, d f=5 / 75, p<.01$ and $F=5.64, d f=3 / 75$, $\mathrm{p}<.01)$. The Duncan test showed that fewer errors were made under the $C$ condition (negative pattern rotates) than under the $B, D$, or $A$ conditions. The $B, D$, and $A$ conditions did not differ from each other. Learning curves for each of the conditions are shown in Fig. 2.

\section{DISCUSSION}

The results demonstrate that the orientation of a stimulus is a variable of some importance in determining discrimination performance. In the rotating conditions, Ss must learn not only to respond or not respond to form or object, but also form or object in several orientations, which, depending on the contours and internal characteristics of the stimuli, would be more or less difficult. Support for this view is evident in the greater number of errors made under the condition in which both positive and negative stimuli rotate from trial to trial.

That the best performance occurred when the negative stimulus rotated and the positive stimulus remained constant was somewhat surprising, although it was consistent with the results of Gardner \& Coates (1965). It was expected that the condition in which neither stimulus rotated would give the fewest errors. An explanation of discrimination learning as basically an excitatory process, i.e., as learning to approach the positive object (Ettlinger, 1960), would predict the same performance when the positive stimulus remains constant regardless of what happens with the negative stimulus. On the contrary, Conditions $\mathrm{A}$ and $\mathrm{C}$ did differ. If $S$ is learning more or less equally to avoid the

Table 2. Errors in pattern discrimination

\begin{tabular}{lcc}
\multicolumn{1}{c}{ CONDITION } & Mn & SD \\
\hline A. Both objects in standard orientation & 110 & 36.18 \\
B. Positive object rotates & 113.6 & 40.67 \\
C. Negative object rotates & 87.3 & 39.45 \\
D. Both objects rotate & 113 & 39.15 \\
\hline
\end{tabular}

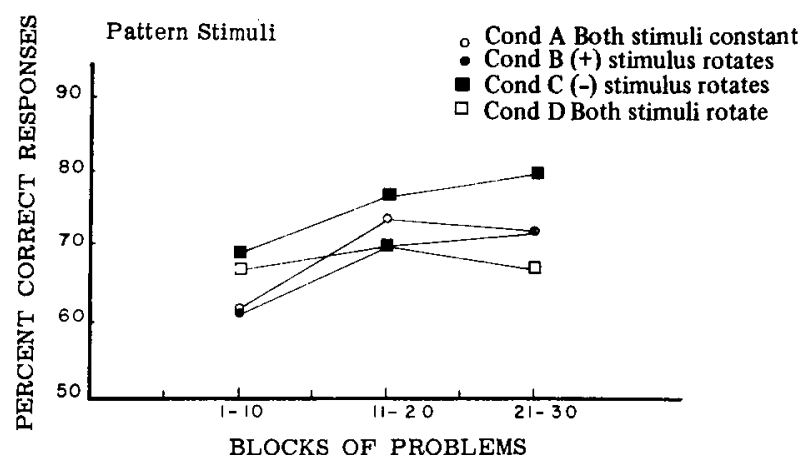

Fig. 2. Mean correct responses for blocks of problems under various stimulus pattern rotation conditions.

negative stimulus and approach the positive stimulus (e.g., Jenkins, 1965), Condition A (both stimuli constant) should produce the best performance. This did not occur. In a similar fashion, an explanation in which $S$ is presumed to learn to avoid the negative stimulus will not explain the data. The results are exactly opposite to this prediction, which would demand that Condition B (positive stimulus rotates) performance be superior to Condition $\mathrm{C}$ (negative stimulus rotates). One might accept the compromise position that response choices in discrimination learning depend on both approach to the positive stimulus and avoidance of the negative stimulus. The relative strength of these tendencies would vary as a function of a number of factors, such as position and object preferences, response perseveration, and response shift tendencies. Then the superiority of the $\mathrm{C}$ condition might be explained in terms of approach to a stable, rewarded positive stimulus and avoidance of the less stable, nonrewarded negative stimulus which becomes more background, less emphatic, and less figural.

\section{References}

ETTLINGER, G. Discrimination learning theory: excitatory vs. inhibitory tendencies in monkeys. Quart. J. exp. Psychol, 1960, 12, 4144.

GARDNER, R. A., \& COATE, W. B. Reward versus nonreward in a simultaneous discrimination. J. exp. Psychol, 1965, 69, 579-582.

JENKINS, H. M. Generalization gradients and the concept of inhibition. In D. Mostofsky (Ed.), Stimulus generalization. Stanford: Stanford University Press, 1965.

SUTHERLAND, N. S. Shape discrimination in animals. Experimental Psychology Society Monograph. No. 1. Cambridge, England: W. Heffer \& Sons, 1961.

Note

1. This investigation was supported by NIMH Grant M-1215. 